\title{
Transmission Congestion Management by using Lmp Method in De-Regulated Electricity Market

\author{
V.L.PhaniBhushan, GummadiSrinivasaRao
}

\begin{abstract}
The power transmission network has the problem of management due to congestion in the open access system. Power flow due to transactions in transmission lines can cause overloads. This condition is known as congestion. There are several alternative methods for congestion management which are suitable for different electricity markets. In this paper the Locational Marginal Pricing (LMP) method is discussed for an assessment of transmission congestion management and results are obtained to manage the transmission congestion such as redispatching existing generators outside the congested area to supply power to the customer. The primal dual IP algorithm is used to calculate the LMP's and congestion cost values. The proposed work has been implemented on a 14-bus test system to illustrate the advantages and disadvantages of this method
\end{abstract}

Index Terms - Congestion management, LMP, Interior point method.

\section{INTRODUCTION}

In the open access environment, consumers are choosing their provider for electric energy. In other way, consumers will have an alternative way in buying electricity for fulfilling their needs. Transmission lines in deregulated environment cause overloads due to power flow transactions. This condition is known as congestion. Owing to contravention of system working confines congestion take place in the transmission lines. Due to loop flow problems, generation or power grid outages and also increase in power demand congestion may occur. Now here there are two types of systems i.e. vertically bundled and unbundled systems. The bundled system is somewhat simple to handle the congestion as judge against to unbundled system. In which generation, transmission and in distribution systems are handled by one utility system. Nowadays, in competitive electricity markets the handling of congestion is little bit more complex which leads to a number of troubles.

The congestion management is done in pool and bi-lateral markets by using LMP method. LMP is also known as nodal pricing which is useful to manage congestion in Independent System Operator (ISO). In LMP, the price to transmit power from starting point to ending point replicates the marginal cost of such transmission service supplier. Now here, the transmission service is scheduled by transmission provider. Financial Transmission Right (FTR) will act as a border to the financial entitlements on the congestion charges occurred during congestion management in a specified

Revised Manuscript Received on 16 September, 2019.

V.L.PhaniBhushan, PG Student, Electrical and Electronics Engineering, V.R. Siddhartha Engineering College(Autonomous), Vijayawada, A.P. India. (Email: phanibhushan295@gmail.com)

Dr.GummadiSrinivasaRao, Associate Professor, Electrical and Electronics Engineering, V.R. Siddhartha Engineering College(Autonomous),Vijayawada,A.P.,India.(Email:vasu1in@vrsiddharth a.ac.in) transmission path. Moreover, the LMP prices are calculated and analyzed. In this paper the results are discussed based on locational marginal pricing (LMP) and presented an assessment of transmission congestion management in restructured electricity marketplace. The assessment is done on a 14-bus system to illustrate the advantages and disadvantages of LMP method.

\section{OVERVIEW OF TRANSMISSION CONGESTION MANAGEMENT}

Transmission Congestion management plays an important role in deregulated environment. Transmission congestion occurs when the power system violates the operating conditions like independent transactions in the transmission lines, faults in the line, sudden shutdown of generators, mismatch between demand and supply, domination of prices in some areas and damages in system components. Owing to, the new contracts are decreased; infeasibility in the system takes place. As a result, ISO faces the complexity in managing the congestion. Hence Congestion is to be handled through congestion charges and wheeling charges such they are found in a power trading exchange.

\section{LOCATIONAL MARGINAL PRICING (LMP)}

In some developed countries, LMP is adapted as the pricing method to handle the congestion. This price is also known as locational marginal price (LMP). Locational marginal pricing is a path for the wholesale electric energy prices to locate the value of electric energy at different locations such that considering the load, generation and the physical limits of the transmission system.

There are a minimum of four ways to calculate locational marginal prices. They are:-

i) The operation of the system can be done optimally"before" $1 \mathrm{MW}$ increase and "after" $1 \mathrm{MW}$ increase.Theadditional cost of operating the system optimally after delivering the additional MW is called as LMP. This method is highly not practical.

ii) The LMPs are to be found by Langrangian multipliers from the optimal power flow (OPF) computations are to be done to compute the optimal dispatch.

iii) The LMP method is also known as nodal pricing suchthat it can be calculated in two ways, 
i) If there are no transmission constraints between two nodes the nodes are taken as one common zone and calculate the LMPs in that zone and they are same for both the nodes.

ii) If transmission constraints are present in between the two nodes the LMPs will be calculated for both the nodes separately.

iii) The LMPs can also be obtained by a "transposed Jacobian" matrix solution at a given operating point, and these LMPs are used to manage the congestion in any system with respective transmission constraints. Here, in LMP calculation losses are not considered without transmission constraints. Based on generator bid resources, the generators need to re-dispatch in a system.

The algorithm of market dispatch in without system losses and system limits may be given as:

$$
\begin{aligned}
& \min \sum_{i=1}^{N_{G}} \mathrm{CGi}\left(\mathrm{P}_{\mathrm{Gi}}\right) \\
& \text { and is subjected to, }
\end{aligned}
$$

$$
\sum_{\substack{i=1 \\ 0 \leq \mathrm{P}_{\mathrm{Gi}} \leq \mathrm{P}_{\mathrm{Gi}}^{\max }}}^{N_{G}} \mathrm{P}_{\mathrm{Gi}}=\sum_{j=1}^{N_{D}} \mathrm{P}_{\mathrm{Di}}
$$

Here CGi $\left(\mathrm{P}_{\mathrm{Gi}}\right)=\alpha_{\mathrm{Gi}} \mathrm{P}_{\mathrm{Gi}}=$ Bid-based cost function of generator $\mathrm{i}$.

Here,

$\alpha_{\mathrm{Gi}}=$ ith generator competitive price

$\mathrm{P}_{\mathrm{Gi}}=\mathrm{ith}$ generator Power supplied

$\mathrm{N}_{\mathrm{G}}=$ Total generating units

$\mathrm{N}_{\mathrm{D}}=$ Number of loads

$\mathrm{P}_{\mathrm{Gi}}{ }^{\max }=$ Maximum generator capacity of generator $\mathrm{i}$

Moreover, here in equation (2) stands for the total generation have to be equivalent to the total claim of power. The constraint on which is present in equation (3) determines about the generation capacity.

From the information obtained from the above equations, the SO (System Operator) estimate the possible power flows within the system such that it will have the supply and demand position of all market contributors. When congestion occurs, the security-constrained OPF (Optimal Power Flow) problem (1)-(3) and the inequality constraint can be calculated by the following equation given below:

$$
\left.\mathrm{P}_{\mathrm{kl}}^{0}+\sum_{i=1}^{N_{G}} \mathrm{a}_{\mathrm{kl}, \mathrm{i} \cdot} \cdot \mathrm{P}_{\mathrm{Gi}}{ }^{\text {adj }}-\mathrm{P}_{\mathrm{Gi}}^{0}\right) \leq \mathrm{p}_{\mathrm{kl}}^{\max }
$$

$\mathrm{P}_{\mathrm{kl}}^{0}=$ initial power flow on the constraint line $\mathrm{k}-\mathrm{l}$

$\mathrm{P}_{\mathrm{kl}}{ }^{\max }=$ maximum power flow limit on the constraint

line $\mathrm{k}-1$

$\mathrm{P}^{0}{ }_{\mathrm{Gi}}=$ initial power output for generator $\mathrm{i}$

$\mathrm{P}_{\mathrm{Gi}}^{\text {adj }}=$ final adjustment power output for generator $\mathrm{i}$

$$
\mathrm{N}_{\mathrm{G}}=\text { Number of generator buses }
$$

$\mathrm{a}_{\mathrm{k}, \mathrm{i}}=\mathrm{dc}-\mathrm{PTDF}$, the sensitivity of the flow on line $\mathrm{k}-\mathrm{l}$ to a change in generation at bus $\mathrm{i}$

The added megawatt of power is supplied at every bus, then the incremental generation is calculated for an each marginal generator. The lowest cost and the price can be determined by the following,

$$
\begin{aligned}
& \min \sum_{i=1}^{N_{G}} C_{G i}\left(P_{G i}\right) \\
& \sum_{i=1}^{N_{G}} \Delta \mathrm{P}_{\mathrm{Gi}}=\sum_{j=1}^{N_{D}} \Delta \mathrm{P}_{\mathrm{Dj}} \\
& \Delta \mathrm{P}_{\mathrm{Gi}}^{\min } \leq \Delta \mathrm{P}_{\mathrm{Gi}} \leq \Delta \mathrm{P}_{\mathrm{Gi}} \max \\
& \sum_{i=1}^{N_{G}} a_{k l, i} . \Delta \mathrm{P}_{\mathrm{Gi}}+\sum_{j=1}^{N_{D}} a_{k l, i} . \Delta \mathrm{P}_{\mathrm{Dj}} \leq 0
\end{aligned}
$$

Where,

$\mathrm{C}_{\mathrm{Gi}}\left(\mathrm{P}_{\mathrm{Gi}}\right)=$ ith generator competitive-based Generation cost.

$\Delta \mathrm{P}_{\mathrm{Gi}}=$ Power output change for generator i.

$\Delta \mathrm{P}_{\mathrm{Dj}}=$ Power output change for demand $\mathrm{j}$.

$\mathrm{N}_{\mathrm{G}}=$ No. of marginal generators.

$\mathrm{N}_{\mathrm{D}}=$ No. of load buses.

$\Delta \mathrm{P}_{\mathrm{Gi}}{ }^{\max }=$ Maximum adjustable capacity generation at generator $\mathrm{i}$.

$\Delta \mathrm{P}_{\mathrm{Gi}}{ }^{\mathrm{min}}=$ Minimum adjustable capacity generation at generator $\mathrm{i}$.

$a_{k l, i}=$ The PTDF flow on the line k-l to the change in generation at bus $\mathrm{i}$.

$a_{k l, j}=$ The PTDF flow on the line $\mathrm{k}-1$ to the $\mathrm{j}$ th bus change in demand

By these above equations the LMPs are found by multiplying the SFs and the consequent bid prices of the marginal generators are achieved.

When the increment in electric demand at a particular bus in the network, the marginal cost of providing that increment is replicated by the LMPs.Based on the variation between these values all the energy transactions give congestion charges 


\section{TRACING METHODOLOGY OF CONGESTION}

Generally Power Transfer Distribution Factors (PTDFs) is employed for handling the congestion. This method is used to choose the restriction and re-dispatch due to congestion. The set of network sensitivity factors is given by the PTDF (or) generation shift factor (GSF) to estimate the flow changes to a specific power transfer between two points on the system. The sensitivity of the flow on line $i-j$ to a change in generation at bus $\mathrm{k}$ is represented by PTDF. It is having the following definition:

$$
a_{i-j, k}=\frac{\Delta P_{i-j}}{\Delta P_{G_{k}}}
$$

Where,

$\Delta P_{G_{k}}=\mathrm{i}$ th bus generation change.

$\Delta P_{i-j}=$ Alteration of active Power flow in line $\mathrm{i}-\mathrm{j}$ due to generation change at bus $\mathrm{k}$.

PTDFs are obtained by the DC power flow. This factor is obtained to give the linear prediction of power flow distribution in response to change in generation and also load demand changes. Moreover, these factors can be obtained to predict the maximum power transaction that can be allowed in the transmission line.

By using the depiction of the reactance matrix and the DC estimatation, the PTDF can be found by the following equation,

$$
a_{i-j, k}^{m}=\frac{\Delta P_{i-j}}{\Delta P_{G_{k}}}=\frac{X_{i k}-X_{j k}}{x_{i j}}
$$

Where,

$$
\begin{aligned}
& X_{i k}, X_{j k}=\text { reactance matrix elements. } \\
& x_{i j}=\text { line reactance for the line } \mathrm{i}-\mathrm{j} .
\end{aligned}
$$

Here, in the above expressions the line reactance and the reactance matrix elements are having the buses $\mathrm{i}, \mathrm{j}$ and $\mathrm{k}$. Moreover, by these buses they are having connected to the three lines i-j, j-k, i-k.

\section{LINEAR PROGRAMMING}

Linear Programming is a mathematical model to achieve the optimal output. This is one of the optimization technique in which is used to solve the linear optimal equations. It also consists of linear objective function, subject to inequality and equality conditions. In this the lossless DCOPF optimization problem is formed as a linear programming problem. In this the interior point algorithm is used to solve both the linear and non-linear programming problems to find the (LMP) locational marginal prices and congestion cost prices. In interior point algorithm there are two methods they are the barrier method and the primal dual IP method. method. Due to its efficiency and accuracy the primal dual method is used for solving the non-linear programming problem (NLP). The algorithm for the primal dual IP But in this paper, the analysis is done by using primal dual

method is described below for finding the generation shift factor matrix, LMP and Congestion cost values.

Step 1: Minimize $f(x)$ i.e. $g(x) \geq 0$ with $i=1, \ldots, m$.

Step 2: Introduce slack variables to change all the inequalities into non-negativities.

Step 3: Again minimize $f(x)$ i.e. $g(x)-s=0$ with $s \geq 0$.

Step 4: Minimize $\mathrm{f}(\mathrm{x})-\mu \sum_{i=1}^{m} \log \left(s_{i}\right)$ which is a logarithmic barrier function where $\mathrm{h}(\mathrm{x})-\mathrm{s}=0$.

Step 5: Next incorporate the equality constraints into the objective function using the langrangian multiplier:

$$
\text { Minimize } \mathrm{f}(\mathrm{x})-\mu \sum_{i=1}^{m} \log \left(s_{i}\right)-\mathrm{y}^{\mathrm{T}}(\mathrm{g}(\mathrm{x})-\mathrm{s})
$$

Step 6: Next set all the derivatives equals to zero and rearrange them.

Step 7: Then the Newton's method is utilized to determine the search directions, i.e. $\Delta \mathrm{x}, \Delta \mathrm{s}, \Delta \mathrm{y}$ and perform iterations for the obtained equations.

Step 8: Form generation shift factor matrix $\left(\mathrm{S}_{\mathrm{f}}\right)$ and find $\mathrm{B}$ matrix i.e. (B1, B2...,Bn).

Step 9: Find the LMP and Congestion cost values.

\section{PROPOSED WORK AND RESULTS}

The proposed work is prepared on of IEEE-14 bus test system. This system consists of 20 lines and 14 buses. The Generator data for IEEE-14 bus system are shown in the Table 1.

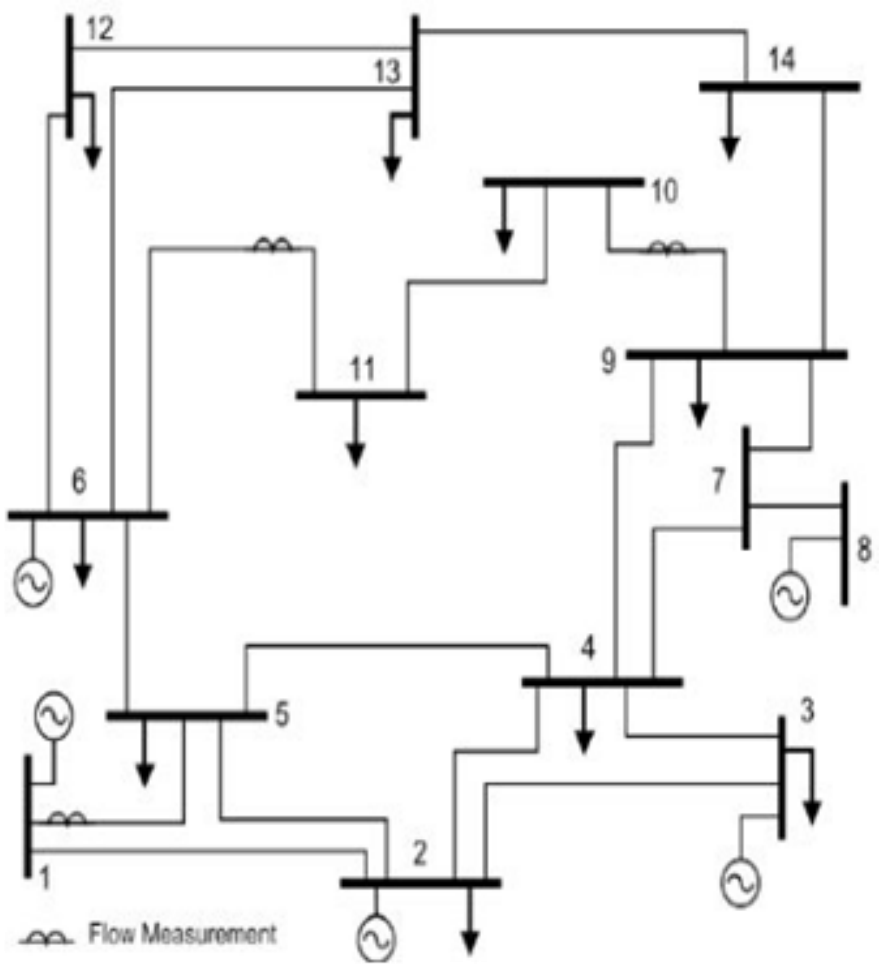

Fig 1: IEEE-14 Bus One line diagram. 
Table 1 Generator Data

\begin{tabular}{|c|c|c|c|c|c|}
\hline $\begin{array}{c}\text { Genco } \\
\text { Numbe } \\
\mathbf{r}\end{array}$ & $\begin{array}{c}\mathbf{P}_{\mathbf{i}}^{\min } \\
(\mathbf{M W})\end{array}$ & $\begin{array}{c}\mathbf{P}_{\mathbf{i}}^{\mathbf{m a x}} \\
\mathbf{( \mathbf { M W } )}\end{array}$ & $\begin{array}{c}\mathbf{a}_{\mathbf{i}} \\
\left.(\mathbf{\$} / \mathbf{M W h r})^{2}\right)\end{array}$ & $\begin{array}{c}\mathbf{b}_{\mathbf{i}} \\
(\mathbf{\$} / \mathbf{M W h r})\end{array}$ & $\begin{array}{c}\mathbf{c}_{\mathbf{i}} \\
\mathbf{( \$ / \mathbf { h r }} \\
\mathbf{)}\end{array}$ \\
\hline $\mathbf{G}_{\mathbf{1}}$ & 10 & 160 & 0.005 & 2.450 & 105.0 \\
\hline $\mathbf{G}_{\mathbf{2}}$ & 20 & 80 & 0.005 & 3.510 & 44.10 \\
\hline $\mathbf{G}_{3}$ & 20 & 50 & 0.005 & 3.890 & 40.60 \\
\hline
\end{tabular}

From the table 1, ai, bi, ci are the cost co-efficients of the generator data. The cost co-efficientsai, bi, ci are taken in ((\$/MWhr) 2, \$/MWhr, \$/hr)). These coefficients are taken to calculate the congestion cost and LMP values.

Simulation is carried out by using MATLAB coding. The interior point algorithm is used to find the LMP values and congestion cost. The following cases are considered for the analysis.

Case1: LMP values before congestion (Normal Condition).

Case2: LMP values after congestion (When Congestion Occurred).

Case 1: LMP values before congestion (Normal condition)

LMP is calculated using DCOPF without losses for the IEEE-14 bus test system in which are shown in the following Table 2 .

Table 2 LMP values before congestion (Normal Condition)

\begin{tabular}{|c|c|c|}
\hline S.No & Bus No. & $\begin{array}{c}\text { LMP } \\
(\$ / M W h r)\end{array}$ \\
\hline 1 & 1 & 2.6339 \\
\hline 2 & 2 & 2.6339 \\
\hline 3 & 3 & 2.6339 \\
\hline 4 & 4 & 2.6339 \\
\hline 5 & 5 & 2.6339 \\
\hline 6 & 6 & 2.6339 \\
\hline 7 & 7 & 2.6339 \\
\hline 8 & 8 & 2.6339 \\
\hline 9 & 9 & 2.6339 \\
\hline 10 & 10 & 2.6339 \\
\hline 11 & 11 & 2.6339 \\
\hline 12 & 12 & 2.6339 \\
\hline 13 & 13 & 2.6339 \\
\hline 14 & 14 & 2.6339 \\
\hline
\end{tabular}

Case 2: LMP values after congestion. (When Congestion Occurred)

LMPs are calculated using DC OPF without loss for the IEEE-14 Bus System when congestion occurred by calculating line limits. From the table 2 and 3, it is observed that, the LMP values in normal condition are same at all the buses. But in the case of when congestion occurred, the LMP values are varying from location to location.
Table 3 LMP values when congestion occurred(After Congestion)

\begin{tabular}{|c|c|c|}
\hline S.No & Bus No. & $\begin{array}{c}\text { LMP } \\
(\$ / \text { MWh })\end{array}$ \\
\hline 1 & 1 & 2.1828 \\
\hline 2 & 2 & 4.6251 \\
\hline 3 & 3 & 4.3656 \\
\hline 4 & 4 & 4.1414 \\
\hline 5 & 5 & 3.9513 \\
\hline 6 & 6 & 4.3656 \\
\hline 7 & 7 & 4.2023 \\
\hline 8 & 8 & 4.2023 \\
\hline 9 & 9 & 4.2343 \\
\hline 10 & 10 & 4.2576 \\
\hline 11 & 11 & 4.3107 \\
\hline 12 & 12 & 4.3552 \\
\hline 13 & 13 & 4.3471 \\
\hline 14 & 14 & 4.2836 \\
\hline
\end{tabular}

\section{CONCLUSION}

In this paper, the locational marginal pricing plays a prominent role in deregulated electricity markets. The interior point method is employed to calculate LMP's and congestion cost values and analyzed for congestion management. This work can be extended further by considering the power loss as constraint and also considering the line stability limits along with thermal limits.

\section{REFERENCES}

1. Shahidehpour M, Yamin H, Li Z, "Market operations in electric power systems," New York: Wiley; 2002.

2. Kirschen DS, Strbac G, "Fundamentals of power system economics," Wiley; 2004.

3. A. J. Wood, B. F. Wollenberg, "Power Generation, Operation and Control (second edition)," John Wiley\&Sons, New York, 1996 